\title{
Coherence and decoherence in the Harper-Hofstadter model
}

\author{
Q.-Y. Liang, ${ }^{1}$ D. Trypogeorgos, ${ }^{1,2}$ A. Valdés-Curiel, ${ }^{1}$ J. Tao,${ }^{1}$ M. Zhao, ${ }^{1}$ and I. B. Spielman $\odot^{1, *}$ \\ ${ }^{1}$ Joint Quantum Institute, National Institute of Standards and Technology, and University of Maryland, Gaithersburg, Maryland 20899, USA \\ ${ }^{2}$ CNR Nanotec, Institute of Nanotechnology, via Monteroni, 73100, Lecce, Italy
}

(Received 9 December 2020; revised 19 March 2021; accepted 22 March 2021; published 19 April 2021)

\begin{abstract}
We quantum simulated the 2D Harper-Hofstadter $(\mathrm{HH})$ lattice model in a highly elongated tube geometrythree sites in circumference-using an atomic Bose-Einstein condensate. In addition to the usual transverse (out-of-plane) magnetic flux, piercing the surface of the tube, we threaded a longitudinal flux $\Phi_{\mathrm{L}}$ down the axis of the tube. This geometry evokes an Aharonov-Bohm interferometer, where noise in $\Phi_{\mathrm{L}}$ would readily decohere the interference present in trajectories encircling the tube. We observe this behavior only when transverse flux is a rational fraction of the flux quantum and remarkably find that for irrational fractions the decoherence is absent. Furthermore, at rational values of transverse flux, we show that the time evolution averaged over the noisy longitudinal flux matches the time evolution at nearby irrational fluxes. Thus, the appealing intuitive picture of an Aharonov-Bohm interferometer is insufficient. Instead, we quantitatively explain our observations by transforming the $\mathrm{HH}$ model into a collection of momentum-space Aubry-André models.
\end{abstract}

DOI: 10.1103/PhysRevResearch.3.023058

Understanding how and when closed quantum systems lose or retain coherence is a central intellectual and practical question for quantum technologies. For example, modern optical atomic clocks operate in highly optimized decoherence-free subspaces created by using "clock" states [1] that are insensitive to the environment, as well as using lasers at magic wavelengths and polarizations that give only common mode energy shifts. In rare cases, such as collisional narrowing [2] or environment assisted tunneling [3], random processes can enhance coherence. Here we add to this list the quasiperiodic lattice described by the Harper-Hofstadter $(\mathrm{HH})$ model $[4,5]$ in a highly-elongated tube geometry-a 1D quasicrystal-by showing that the dynamics can be made immune to environmental noise.

Ultracold atomic gases in optical lattices can mimic Aharonov-Bohm $(\mathrm{AB})$ phase factors using the optical phase of interfering laser beams [6,7]. Even in units of the magnetic flux quantum $\Phi_{0}=h / q$, these systems realize large tunable magnetic fluxes $\Phi=a^{2} B / \Phi_{0}$ per lattice plaquette, where $h$, $q, a$, and $B$ denote Planck's constant, charge, lattice constant, and a uniform magnetic field, respectively. Planar geometries [8-10], narrow Hall ribbons [11,12], and even tubes [13,14] have been realized in experiment. In the tube geometry, the longitudinal flux $\Phi_{\mathrm{L}}$ threading the tube has significant physical consequences $[15,16]$. For example, adiabatically ramping $\Phi_{\mathrm{L}}$ by one flux quantum would drive one cycle of Laughlin's

\footnotetext{
*ian.spielman@nist.gov; http://ultracold.jqi.umd.edu

Published by the American Physical Society under the terms of the Creative Commons Attribution 4.0 International license. Further distribution of this work must maintain attribution to the author(s) and the published article's title, journal citation, and DOI.
}

topological charge pump [17] that can probe both noninteracting and many-body topological systems [18].

The HH model was initially formulated to describe electrons moving in a $2 \mathrm{D}$ crystalline lattice with a transverse magnetic field; in terms of the $\mathrm{AB}$ phase $\Phi$ the $\mathrm{HH}$ model is

$$
\begin{aligned}
\hat{H}= & -J_{\mathrm{s}} \sum_{m, n} e^{i(2 \pi \Phi n+\phi)}|m+1, n\rangle\langle m, n| \\
& -J_{x} \sum_{m, n}|m, n+1\rangle\langle m, n|+\text { H.c. }
\end{aligned}
$$

For isotropic tunneling $J_{\mathrm{s}}=J_{x}$, the resulting "Hofstadter butterfly" energy spectrum was one of the first quantum fractals ever predicted [5]. In planar geometries a uniform Peierls phase $\phi$ has no physical consequence; however, for a tube $M$ sites in circumference $[M=3$ depicted in Fig. 1(a)], the uniform Peierls phase contributes $M \phi /(2 \pi)$ to the longitudinal flux $\Phi_{\mathrm{L}}$ [19]. Using the synthetic dimension approach $[11,12,20]$, we assembled our 2D lattice by combining the sites of a 1D optical lattice with three internal atomic states to respectively define the longitudinal $\left(\mathbf{e}_{x}\right)$ and azimuthal $\left(\mathbf{e}_{\mathrm{s}}\right)$ directions of our tube. We performed interference experiments [Fig. 1(a)] akin to AB interferometers: Particles prepared at site $m=1$ along $\mathbf{e}_{\mathrm{s}}$, but extended along $\mathbf{e}_{x}$, were released and potentially interfered as they rapidly encircled the tube.

In this Letter, we report three key observations summarized in Fig. 1(b), where $\langle\cdot\rangle$ denotes the average over $\phi \in[0,2 \pi)$, and $\langle\cdot\rangle_{t}$ marks the time average. (i) For rational transverse flux $\Phi=P / Q$ (expressed in reduced form), the time-evolving population in each $m$ site depends strongly on $\phi$ and therefore exhibits large uncertainties, as one would expect for an $\mathrm{AB}$ interferometer. (ii) This dependence decreases with increasing $Q$ and vanishes for irrational $\Phi$. (iii) The $\phi$-averaged dynamics at rational $\Phi$ are equal to those at nearby irrational 


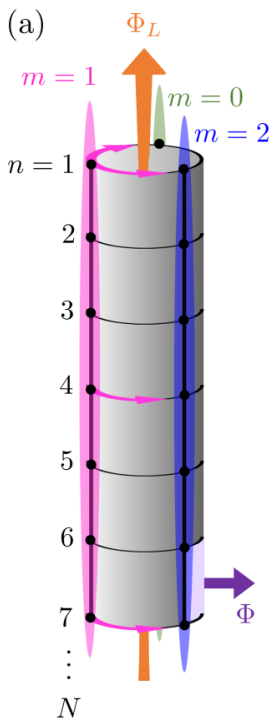

(b) Fractional population, $\left\langle P_{m=1}\right\rangle$

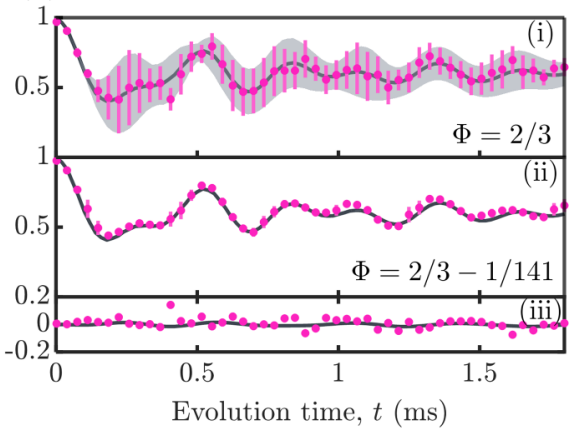

(c) $\sum_{m}\left\langle\operatorname{var}\left[P_{m}\right]\right\rangle_{t} /\left\langle\left\langle P_{m}\right\rangle^{2}\right\rangle_{t}$ $2 / 3$

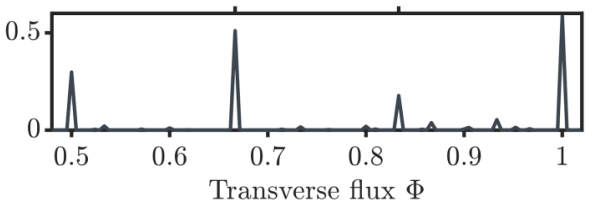

(d)

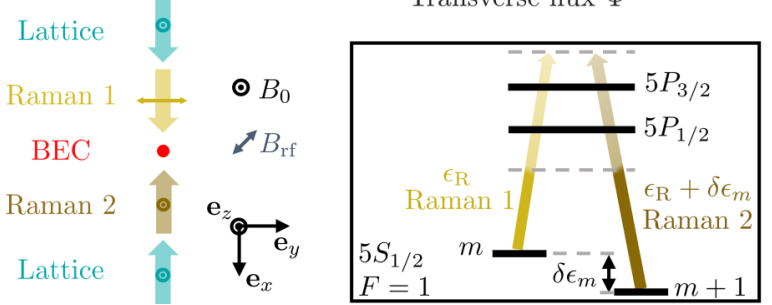

FIG. 1. Schematic and summary of results. (a) The HH model in the tube geometry. (b) Average fractional population $\left\langle P_{m=1}\right\rangle$ at $\Phi=2 / 3$ (i) and $\Phi=2 / 3-1 / 141$ (ii), along with their difference (iii). The pink uncertainty bars denote the standard deviation from the mean across 14 to 22 experimental runs and result from the sensitivity to $\phi$ along with technical noise from: variations in magnetic field, Raman coupling strength, and rf field strength, along with statistical detection uncertainties. The dark blue curves and error bands plot our numerical simulations. (c) Numerically simulated phase noise sensitivity quantified by the normalized variance time averaged over the range $[0,1.8] \mathrm{ms}$. (d) Raman coupling scheme and schematic of experimental setup. The optical lattice beam was retroreflected forming a standing wave. The Raman beams were orthogonally polarized and collinear with the lattice beams.

$\Phi$. In all cases, our numerical simulations are in excellent agreement with our observations. While we experimentally probed $\Phi$ near $2 / 3$, these observations are generalized by the numerical simulation shown in Fig. 1(c) that plots the time-averaged sensitivity to $\phi$; this curve is approximated by an everywhere discontinuous Thomae-like function [21]. Our system's spatial extent $w$ limits the degree to which $\Phi$ can be distinguished to $\approx a / w$, broadening the otherwise singular peaks.

Implementation. We performed these experiments using ${ }^{87} \mathrm{Rb}$ BECs in the $\left|5 S_{1 / 2}, F=1\right\rangle$ electronic ground state manifold in a crossed optical dipole trap [22]. The longitudinal Thomas-Fermi radius $R_{\mathrm{TF}}=11.5(5) \mu \mathrm{m}$ was obtained both by direct in situ imaging and mean-field driven expansion [23]. We used continuous dynamical decoupling (CDD) to eliminate the magnetic field sensitivity [24,25] of this manifold's three $\left|m_{F}\right\rangle$ states, giving three dressed $|m\rangle$ states that served as sites along $\mathbf{e}_{\mathrm{s}}$, with $m=0,1,2$. Our experiments took place in a $B_{0} \approx 31.47 \mathrm{G}$ bias field, along with a resonant $22.1 \mathrm{MHz}$ radio-frequency (rf) magnetic field $B_{\mathrm{rf}}$ with $150 \mathrm{kHz}$ Rabi frequency for CDD. The resulting energy differences $\delta \epsilon_{m} \equiv \epsilon_{m}-\epsilon_{m+1}$ in the CDD basis were $\left(\delta \epsilon_{0}, \delta \epsilon_{1}, \delta \epsilon_{2}\right)=h \times(-308.3,118.6,189.7) \mathrm{kHz}$.

A retroreflected $\lambda_{\mathrm{L}}=532.008(5) \mathrm{nm}$ laser beam along $\mathbf{e}_{x}$ defined our tube's longitudinal sites $|n\rangle$ with spacing $a=$ $\lambda_{\mathrm{L}} / 2$, and the $V=5.0(1) E_{\mathrm{L}}$ lattice depth set the tunneling matrix element $J_{x}=0.066(2) E_{\mathrm{L}}$. Here, $E_{\mathrm{L}}=\hbar^{2} k_{\mathrm{L}}^{2} /\left(2 M_{a}\right)$ and $k_{\mathrm{L}}=2 \pi / \lambda_{\mathrm{L}}$ are the single-photon recoil energy and wave vector, respectively, for atoms with mass $M_{a}$. A pair of Raman laser beams [Fig. 1(d)] resonantly coupled the $|m\rangle$ states with equal strength $\Omega_{\mathrm{R}}=0.296(6) E_{\mathrm{L}}$, providing hopping $J_{\mathrm{s}}=0.111(3) E_{\mathrm{L}}$ along $\mathbf{e}_{\mathrm{s}}$. Raman 2 contained three frequency components $\epsilon_{\mathrm{R}}+\delta \epsilon_{m}$ in the CDD basis to realize the cylindrical geometry, and $\epsilon_{\mathrm{R}}+\delta \epsilon_{2}$ was removed to realize a planar geometry. Because $|m=2\rangle$ was Raman coupled to $|m=0\rangle$, we adopt periodic labels, i.e., $|m\rangle \equiv|\bmod (m, 3)\rangle$. Since $\Phi=$ $k_{\mathrm{R}} / k_{\mathrm{L}}$ [26], with Raman recoil wave vector $k_{\mathrm{R}}=2 \pi / \lambda_{\mathrm{R}}$, we tuned $\Phi=2 / 3+\Delta \Phi$ by varying the Raman wavelength $\lambda_{\mathrm{R}}$ from 770.94(1) $\mathrm{nm}(\Delta \Phi=2 / 87)$ to $806.46(1) \mathrm{nm}(\Delta \Phi=$ $-1 / 141)$. The tuning range was limited by the increasing power requirement as the detuning from the excited states increased. Each experimental run randomly sampled a Peierls phase $\phi$ uniformly distributed from 0 to $2 \pi$, by phase shifting the CDD rf field $B_{\text {rf }}$ [27].

We began with BECs in $|m=1\rangle$, adiabatically loaded into the ground state of the optical lattice, and initiated dynamics by abruptly introducing $J_{\mathrm{s}}$ for a time $t$ up to $1.8 \mathrm{~ms}$, when the lattice, Raman, and dipole trap lasers were simultaneously extinguished. During the subsequent $21 \mathrm{~ms}$ time of flight (TOF) we applied a magnetic field gradient to spatially separate atoms in the three $|m\rangle$ states. We then used absorption imaging to detect the resulting density distribution, yielding the longitudinal momentum distributions of each $|m\rangle$ state.

Model. We quantitatively analyze our experiment by Fourier transforming the $\mathrm{HH}$ Hamiltonian along $\mathbf{e}_{x}$ giving $\hat{H}=\sum_{q_{0}} \hat{H}_{\mathrm{AA}}\left(q_{0}\right)$ with

$$
\begin{aligned}
\hat{H}_{\mathrm{AA}}\left(q_{0}\right)= & -2 J_{x} \sum_{j} \cos \left[2 \pi\left(j \Phi+\frac{q_{0}}{2 \hbar k_{\mathrm{L}}}\right)\right]|j\rangle\langle j| \\
& -J_{\mathrm{s}}\left(e^{i \phi} \sum_{j}|j+1\rangle\langle j|+\text { H.c. }\right),
\end{aligned}
$$

labeled by crystal momentum $q_{0}$. Each $\hat{H}_{\mathrm{AA}}\left(q_{0}\right)$ is a realization of the 1D Aubry-Andrey (AA) lattice [29] with nearest-neighbor hopping strength $J_{\mathrm{s}}$, sinusoidal potential with depth $4 J_{x}$, and phase set by $q_{0}$. The sites of this AA lattice $|j\rangle \equiv\left|m_{0}+j, q_{0}+j \Phi \times 2 \hbar k_{\mathrm{L}}\right\rangle$ are labeled by azimuthal site index $m$ along with longitudinal crystal momentum q. As shown in Fig. 2(a), the sinusoidal potential originates from Raman transitions changing the crystal momentum by $2 \hbar k_{\mathrm{L}} \Phi$ as $m$ is incremented, in effect sampling the lowest band of the longitudinal lattice. For rational $\Phi$, each $\hat{H}_{\mathrm{AA}}\left(q_{0}\right)$ describes a ring [Fig. 2(b)] of size $N_{\mathrm{AA}}=$ $\operatorname{LCM}(M, Q)$ (LCM denotes the least common multiple) since $\left|m_{0}+N_{\mathrm{AA}}, q_{0}+N_{\mathrm{AA}} \Phi \times 2 \hbar k_{\mathrm{L}}\right\rangle$ coincides with the initial state $\left|m_{0}, q_{0}\right\rangle$. For irrational $\Phi$ (incommensurate potential) the 

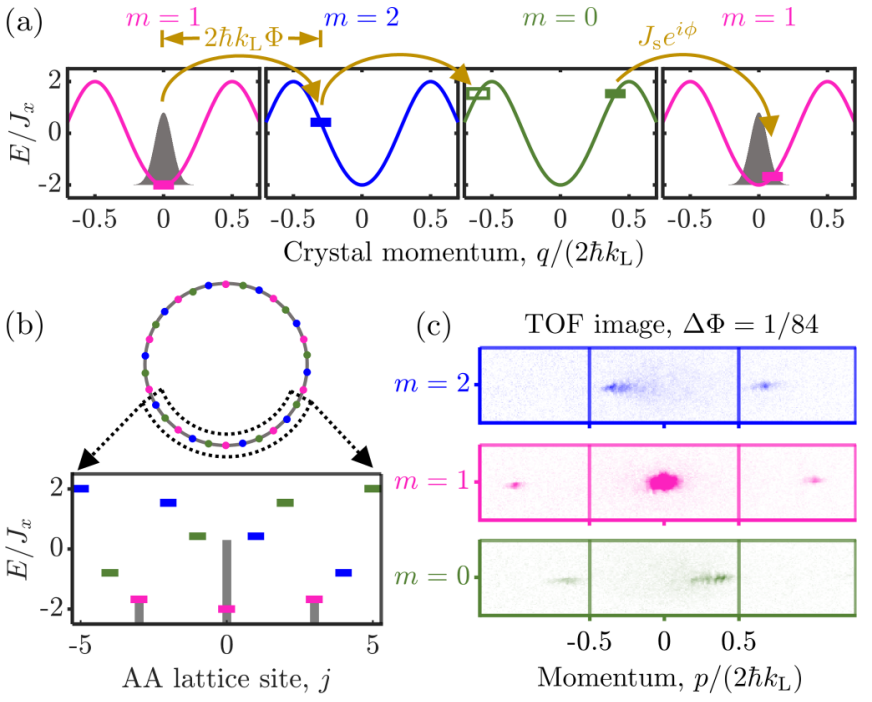

FIG. 2. Momentum space AA model. (a) Lowest band of the longitudinal lattice. The filled gray curve represents a Gaussian wave packet of width $0.064 \times 2 \hbar k_{\mathrm{L}}$. The hollow and solid green rectangles, horizontally spaced by $2 \hbar k_{\mathrm{L}}$, are equivalent points. The arrows mark Raman-induced coupling starting in $|j=0\rangle=$ $\left|m_{0}=1, q_{0}=0\right\rangle$ with $\Delta \Phi=1 / 30$. (b) AA ring corresponding to (a). The bottom panel zooms into the section $-5 \leqslant j \leqslant 5$. The rectangles marking the on-site energy resulting from sampling the dispersion in (a), are colored in accordance to their $|m\rangle$ state, and the gray bars indicate the discretely sampled Gaussian wave packet. (c) TOF data taken at $t=1.506 \mathrm{~ms}$ show the momentum distribution associated with each $|m\rangle$ site. Each crystal momentum state of the longitudinal lattice consists of momentum states that are imaged as horizontally spaced diffraction orders with spacing $2 \hbar k_{\mathrm{L}}$. The synthetic lattice sites are resolved vertically; the diffraction orders in these sites are shifted by $2 \hbar k_{\mathrm{L}} \Phi$ for each synthetic hopping event as expected from (a).

AA model is $1 \mathrm{D}$ quasicrystal with a prototypical metal $\left(J_{\mathrm{S}}>\right.$ $\left.J_{x}\right)$ to insulator $\left(J_{\mathrm{S}}<J_{x}\right)$ transition [30,31], and at criticality $J_{\mathrm{s}}=J_{x}$ it is a quantum fractal, showing features of quantum chaos [32].

We focus on the case relevant to our experiment, with $M=$ 3 and initial site $m=1$. Given the BEC's RMS spatial extent $w \approx 0.46 R_{\mathrm{TF}}=20(1) a$ (Appendix A), the corresponding momentum-space density distribution had RMS width $w_{q} \approx$ $\hbar /(2 w)=4.0(2) \times 10^{-3} \times 2 \hbar k_{\mathrm{L}}$, schematically indicated by the gray Gaussian in the left panel. The azimuthal tunneling from Raman coupling induces transitions (tan arrows) that change the crystal momentum by $2 \hbar k_{\mathrm{L}} \Phi$, as well as imparting the phase $\phi$. Together with $\Phi$, the momentum space width $w_{q}$ specifies the initial occupation of AA lattice sites: Every third AA site potentially samples the initial wave packet until $3 j|\Delta \Phi| \times 2 \hbar k_{\mathrm{L}} \gtrsim w_{q}$ when the site's crystal momentum falls outside the initial wave packet. A representative AA lattice is shown in Fig. 2(b) where the gray bars result from sampling the filled gray curve in Fig. 2(a). In the AA model's metallic phase, where our experiments took place, amplitude ballistically expands from each initially occupied site, while in the insulating phase it remains exponentially localized near each initial site [31]. When $3|\Delta \Phi| \times 2 \hbar k_{\mathrm{L}} \ll w_{q}$, the AA ring contains multiple initially occupied sites; as the system evolves, amplitude originating in different sites can overlap and interfere. In contrast, for $3|\Delta \Phi| \times 2 \hbar k_{\mathrm{L}} \gg w_{q}$, interference is absent due to lack of initial adjacent occupancy. Any interference depends strongly on $\phi$, which appears in the tunneling term of Eq. (2). We quantify the degree of interference by the normalized variance $\operatorname{var}\left[P_{m}(t)\right] /\left\langle P_{m}(t)\right\rangle^{2}$, which is proportional to $\sum_{j \neq 0} S\left(3 \Delta \Phi j \times 2 \hbar k_{\mathrm{L}}\right)$, for $3|\Delta \Phi| \times 2 \hbar k_{\mathrm{L}} \gtrsim$ $w_{q}$ [Eq. (D8)]. $S(\delta q) \equiv\left|\int d q \psi^{*}(q+\delta q) \psi(q)\right|^{2}$ is the static structure factor, and $\psi(q)$ is the momentum-space wave function.

Discussion. Figure 3 summarizes our data for three values of $\Delta \Phi$ and compares the experimental data with our numerical modeling using parameters obtained from fits to the data in Fig. 1(b). These parameters are within the uncertainties (Appendix C) of independent calibrations. To achieve quantitative agreement with our data, we include a phenomenological dephasing parameter obtained from fitting the decaying sinusoid of Fig. 3(a). The top row depicts the AA lattice along with the initial state (gray bars), showing the transition from multiple sources for $\Delta \Phi \approx 0$, to a single isolated source for $\Delta \Phi=$ 1/84. In the ribbon case [Fig. 3(a)], the disconnected links in the lattice, resulting from removing the Raman coupling between $|m=0\rangle$ and $|m=2\rangle$, prevent any potential interference, and the $\phi$ sensitivity vanishes for all transverse flux $\Phi$.

The second row of Fig. 3 plots the three diffraction peaks in the range $-1 / 2<q /\left(2 \hbar k_{\mathrm{L}}\right)<1 / 2$, derived from our TOF data [see Fig. 2(c) for example]. When $\Delta \Phi$ was small enough to allow interference between atoms originating from different AA lattice sites [Figs. 3(b) and 3(c)], the corresponding crystal momentum difference between different paths to the same order is too small to be resolved. When $3|\Delta \Phi| \times 2 \hbar k_{\mathrm{L}} \gg w_{q}$, the momentum difference became resolvable, causing each order in the TOF image to fragment into multiple overlapping suborders. In the ribbon case, only three orders are present irrespective of $\Delta \Phi$.

The third row of Fig. 3 compares the observed fractional population in the initial state $P_{m=1}$ for individual measurements without averaging (top) with the prediction of our model (bottom). The experimental data is highly variable only for small $\Delta \Phi$ with a manifestly non-Gaussian distribution. The noisy dynamics begin at $t \approx \pi \hbar /\left(3 J_{\mathrm{s}}\right)=0.185 \mathrm{~ms}$ when population was significantly transferred out of the initial state, and interference became possible [33]. The numerical model fully captures the observed spread of data, its time dependence, and even the non-Gaussian distribution, including singular features, i.e., caustics [34]. The ribbon case lacks interference and exhibits only technical noise.

The time-averaged normalized variance [Fig. 4(a)] exhibits a sharp peak at $\Phi=2 / 3$ for the tube geometry, despite being featureless for the ribbon geometry. The measured time dependence of the normalized variance, shown in Fig. 4(b), is peaked at $\Delta \Phi=0$ for all times but with variable amplitude. In both cases, the numerical calculations [Fig. 4(c), and solid curve in Fig. 4(a)] are nearly indistinguishable from the data. Lastly, the $\phi$-averaged time evolution for both experiment [Fig. 4(d)] and numerics [Fig. 4(e)] shows no feature at $\Delta \Phi=$ 0 where the noise feature is maximal in Figs. 4(b) and 4(c). This can be directly understood with the $\mathrm{HH}$ model which, by a suitable change of $\phi$, transforms site $n$ to $n=0$ [Eq. (1)]. For 
(a) $\Delta \Phi=0$, Ribbon
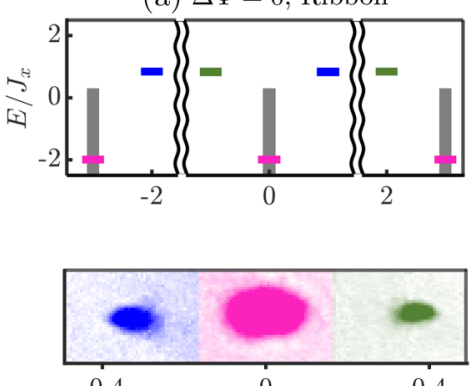

0.4 (b) $\Delta \Phi=0$, Tube

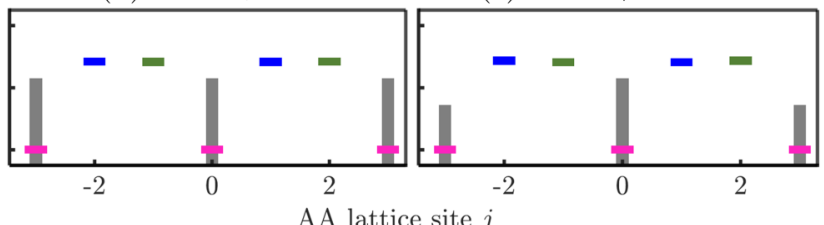

A lattice site (d) $\Delta \Phi=1 / 84$, Tube

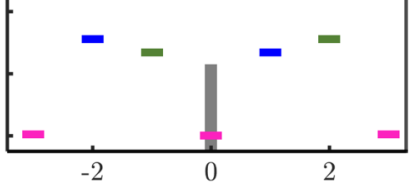

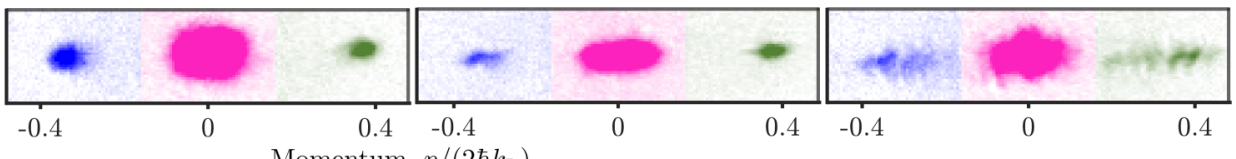

Momentum, $p /\left(2 \hbar k_{\mathrm{L}}\right)$
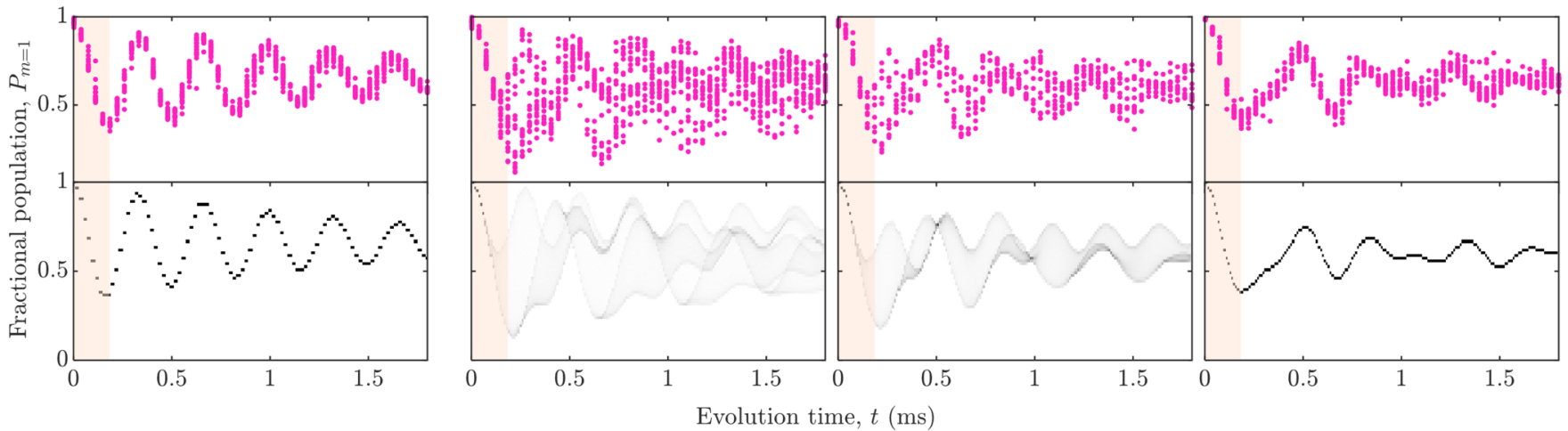

FIG. 3. Representative data. Each column depicts a value of $\Delta \Phi$, from 0 (showing significant $\phi$ dependence) to $1 / 84$ (showing negligible $\phi$ dependence), along with a ribbon geometry as a control case. The top row depicts representative AA lattices $|j=0\rangle=\left|m_{0}=1, q_{0}=0\right\rangle$ for each $\Delta \Phi$, along with the initial wave packets calculated from $R_{\mathrm{TF}}=11.5 \mu \mathrm{m}$ (gray bars). The AA lattice schematic for $\Delta \Phi=0$ was calculated with $\Delta \Phi=1 / 10000$ since the AA ring only has three sites at $\Delta \Phi=0$. The middle row shows TOF data for each case measured at $t=1.506 \mathrm{~ms}$. Since diffraction orders outside $q /\left(2 \hbar k_{\mathrm{L}}\right) \in[-1 / 2,1 / 2)$ replicate those inside, with amplitude governed by the Wannier orbitals of the longitudinal lattice [28], we focus on the regime inside. The bottom row plots the time evolution of single experimental run data (top) and histogram of the predicted trajectories for all $\phi$ (bottom). The shaded regions mark $t<0.185 \mathrm{~ms}$.

irrational transverse fluxes, the system uniformly samples $\phi$, leading to spatially self-averaged time evolution. Although individual systems at rational flux lack the spatial self-averaging effect, averaging over $\phi$ recovers the uniform sampling in the irrational case, resulting in similar mean time evolution as nearby irrational fluxes (Appendix D 3).

Outlook. The realization of the HH model in the highlyasymmetric geometry proved an ideal testbed of the phenomena in this study, and the choice $\Phi \approx 2 / 3$ minimized the distance between the adjacent initially occupied AA lattice sites for $M=3$. The onset time for noisy dynamics should increase with $M$, and if $M$ were comparable to the longitudinal extent, we would not have observed any phase sensitivity within the experimental time scale.

Our AA model makes additional predictions beyond the experimental observations presented here. At longer evolution times than in our experiments, AA rings in the metallic phase with just one initially occupied site can exhibit long-time interference as amplitude fully encircles the ring. While this work did not address the question of giving $\phi$ explicit time
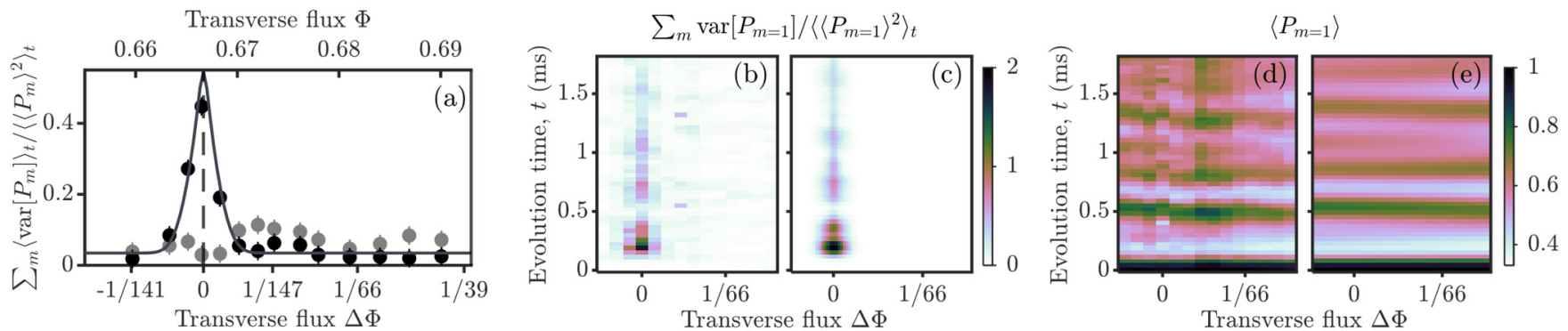

FIG. 4. $\Delta \Phi$ dependance. (a) Phase sensitivity characterized by the time averaged normalized variance is plotted for the fully connected (black) and fragmented (gray) AA rings. The solid line results from the numerical simulation of the full model with $R_{\mathrm{TF}}=11.5 \mu \mathrm{m}$, with baseline shifted by the averaged value of the experimental data away from the peak. Normalized variance (b) and mean time evolution (d) data were linearly interpolated from unequally spaced transverse fluxes probed experimentally. (c) and (e) are their corresponding numerical simulations, respectively. 
dependence, we might expect that the dynamics will be unchanged, provided that both $J_{\mathrm{s}}$ and $J_{x}$ are large compared to the energy shift from the effective electric field $\xi d \phi / d t$, over the extent of the AA localization length $\xi$. If true, this would be a new type of quasidisordered-stabilized decoherence-free subspace.

It is worth noting that spatially $1 \mathrm{D}$ systems, in which interaction and correlation effects become important-a Hall tube like ours - may host exotic topological and magnetic states [35-39]. We hope our study will inspire more research in such systems.

We thank R. P. Anderson for assistance in the early stages of the experiment. We are grateful to Q. Zhou for providing Ref. [31] in advance of submission, E. Tiesinga and P. K. Elgee for careful reading of the paper, as well as H. M. Hurst, E. B. Rozenbaum, and W. D. Phillips for extended discussions. Lastly, we appreciate our interactions with M. Rigol and V. Galitski who convinced us that this result has absolutely nothing to do with quantum chaos. This work was partially supported by the AFOSRs Quantum Matter MURI, NIST, and the NSF (1430094) through the PFC at the JQI.

\section{APPENDIX A: EXPERIMENTAL DETAILS}

Each experiment began with a BEC of ${ }^{87} \mathrm{Rb}$ in a far-detuned crossed dipole trap, with trap frequencies $\left(f_{x}, f_{y}, f_{z}\right) \approx(44,53,159) \mathrm{Hz}$ in $\left|F=1, m_{F}=-1\right\rangle$ sublevel, where $F$ and $m_{F}$ are the total atomic and magnetic angular momentum, respectively. Owing to the central role of the system size in our discussion, we independently determined the longitudinal Thomas-Fermi radius $R_{\mathrm{TF}}$ of the BEC using both TOF and in situ images. We used the Castin-Dum equations [23] to obtain $R_{\mathrm{TF}}=11.6(5) \mu \mathrm{m}$ from TOF images. In addition, digitally refocused in situ images [40,41] gave $R_{\mathrm{TF}}=11.4(6) \mu \mathrm{m}$. Because these two measurements share the same magnification, their uncertainties are correlated and take the average $R_{\mathrm{TF}}=11.5(5) \mu \mathrm{m}$.

The rf field for the dynamical decoupling $B_{\text {rf }}$ [Fig. 1(d) in the main text] was assigned a randomized phase just prior to adiabatically loading the atoms into the $|m=0\rangle$ pseudospin state. This mapped the $\left|m_{F}=-1,+1,0\right\rangle$ magnetic sublevels to the $|m=0,1,2\rangle$ pseudospin states [24], which are insensitive to the magnetic field and facilitate cyclic Raman coupling [42]. The magnetic field $B_{0}$ and $\mathrm{rf}$ strength $\Omega_{\mathrm{rf}}$ were stabilized using the procedure detailed in Sec. A 2. We then applied an rf $\pi$ pulse to prepare the atoms in the $|m=1\rangle$ state. This field was linearly polarized in the direction orthogonal to both $B_{\text {rf }}$ and $B_{0}$. We removed any remaining atoms in $|m=0\rangle$ by transferring them to the $F=2$ hyperfine manifold and blowing them away with a resonant light pulse. Meanwhile, the atoms were adiabatically loaded into the 1D optical lattice with a ramp duration of $200 \mathrm{~ms}$. We then studied dynamics by switched on the Raman coupling for the evolution time $t$, at which point the Raman, optical lattice, and dipole trap beams were suddenly extinguished, initiating TOF.

To achieve the momentum and pseudospin resolved imaging, we reversed the process of loading the atoms into the rf dressed states. Namely, during the $21.3 \mathrm{~ms}$ TOF, we adiabatically ramped down the magnetic field and then ramped
$\Omega_{\text {rf }}$ to zero. As a result, states $|m=0,1,2\rangle$ mapped back to $\left|m_{F}=-1,+1,0\right\rangle$, respectively, which were then separated using the Stern-Gerlach effect with a bias field perpendicular to the lattice beam propagation direction. The absorption image in Fig. 2(b) in the main text does not show the $|m\rangle$ states in the same relative positions as they appeared on our camera $(|m=1\rangle$ and $|m=2\rangle$ states were switched for logical clarity). Additionally, due to the high magnetic field gradient applied, a harmonic potential perpendicular to the bias field stretches $\left|m_{F}=+1\right\rangle$ and compresses $\left|m_{F}=-1\right\rangle$ states by $3 \sim 4 \%$. The optical density profiles of $\left|m_{F}= \pm 1\right\rangle$ sublevels were rescaled such that the distances between the neighboring orders, equal to the two-lattice-photon recoil momenta, were the same for all three sublevels.

\section{Raman setup}

The Raman beams were almost collinear with the counterpropagating optical lattice beams, with the lattice beam bisecting the angle $\beta=0.34(6)^{\circ}$ between the two Raman beams. In this configuration, the transverse flux per lattice plaquette was $\Phi=k_{R} \cos [\beta / 2] / k_{L} \approx k_{R} / k_{L}$. The tiny angle $\beta$ was introduced to avoid retroreflected beams off of common optics. The Raman beams were carefully aligned such that there was no momentum transfer along the transverse direction making the dynamics of our system essentially onedimensional.

We locked the relative phase between Raman beams, the optical lattice beams, and $B_{\text {rf }}$. We detected the beatnote of the two Raman beams in the vicinity of the retro mirror of the optical lattice. The beatnote had two or three frequency components corresponding to the two or three Raman transitions. The $|m=0\rangle \leftrightarrow|m=1\rangle$ transition had the largest intensity and was relatively far from the other transitions, and was therefore chosen as the locking frequency. The local oscillator was taken from the same direct digital synthesized (DDS) signal generator as $B_{\mathrm{rf}}$, such that the rf field for the dynamical decoupling and the Raman beatnote were in phase. We applied feedback to applied to the Raman beam with one frequency component [Raman 1 in Fig. 1(d) in the main text]. In the rotating frame, the energy differences between the two Raman beams were $\delta \epsilon_{m} \equiv \epsilon_{m}-\epsilon_{m+1}$, where $m=0,1,2$. In the laboratory frame, they are $\delta \epsilon_{m}-\epsilon_{\mathrm{rf}}$, so the beatnote frequencies were $\approx 22 \mathrm{MHz}[25]$.

\section{Magnetic field and rf locks}

We then applied two microwave pulses of duration $T_{\mu w}=$ $100 \mu \mathrm{s}$, separated by $1 / 60 \mathrm{~s}$ to partially transfer $\sim 5 \%$ of the atoms to $\left|F=2, m_{F}=-1\right\rangle$ sublevel. At the locking point, the two pulses were blue and red detuned by $1 /\left(2 T_{\mu w}\right)$ from the resonance. The lock point $B_{\text {lock }} \approx 3.137 \mathrm{mT}$ gave an $\mathrm{rf}$ frequency $\omega_{\mathrm{rf}} /(2 \pi)=22.1 \mathrm{MHz}$ resonant with the $\left|F=1, m_{F}=-1\right\rangle \leftrightarrow\left|F=1, m_{F}=0\right\rangle$ transition. The error signal was the imbalance of the two transfers

$$
\epsilon=\frac{O D_{\mathrm{int}}^{(1)}-O \mathrm{OD}_{\mathrm{int}}^{(2)}}{O D_{\mathrm{int}}^{(1)}+O D_{\mathrm{int}}^{(2)}},
$$

where $\mathrm{OD}_{\text {int }}^{(\mathrm{i})}, i=1,2$, was the integrated optical density of the two images. This error signal drove a slow proportional- 
integral-differential (PID) lock that set the magnetic fields in subsequent reputations of the experiment. Our procedure extends earlier work [43], and for further specifics see Ref. [44].

After the atoms were transferred to $|m=0\rangle$ state, we used a similar technique to stabilize the strength of the rf field $\Omega_{\mathrm{rf}}$. The microwave pulses drove the transition with the largest sensitivity to $\Omega_{\mathrm{rf}}$ and the least sensitivity to the $B_{0}$, namely, from $|m=0\rangle$ to the state that asymptotically goes to $\left|F=2, m_{F}=-2\right\rangle$ with decreasing magnetic field.

\section{APPENDIX B: REDUCTION TO HH MODEL}

We start with the full Hamiltonian

$$
\begin{aligned}
\hat{H}_{\text {full }}= & {\left[\frac{\hbar^{2} \hat{k}^{2}}{2 M_{a}}+\frac{V_{\mathrm{L}}}{2} \cos \left(2 k_{L} \hat{x}\right)+V_{\mathrm{ext}}(\hat{x})\right] \otimes \hat{1} } \\
& +\left[\sum_{m} \delta_{m}|m\rangle\langle m|\right] \\
& -\left[\sum_{m} \frac{\Omega_{\mathrm{R}, m}}{2} e^{i\left(2 k_{R} \hat{x}+\phi\right)}|m+1\rangle\langle m|+\text { H.c. }\right],
\end{aligned}
$$

describing the light-matter interaction of our three-state atoms of mass $M_{a}$ including a state independent confining potential $V_{\text {ext }}(\hat{x})$. In our experiment, we measured the $V=5.0(1) E_{\mathrm{L}}$ lattice depth by suddenly applying the lattice potential and fitting the resulting Kapitza-Dirac time evolution [45]. We obtained the Raman coupling strength $\Omega_{\mathrm{R}, m}=\Omega_{\mathrm{R}}=0.296(6) E_{L}$ by separately measuring the Rabi frequencies of each transition and adjusting them to be equal within our uncertainties. This process was repeated each time we changed the wavelength of the titanium sapphire laser producing the Raman laser beams. The parameters $\delta_{m}=0.00(2) E_{\mathrm{L}}$ describe detuning from Raman resonance. The stated uncertainties include the variation across the measured transverse fluxes.

The ground-band behavior of $\hat{H}_{\text {full }}$ can be approximated by the tight-binding $\mathrm{HH}$ Hamiltonian

$$
\begin{aligned}
\hat{H}(\phi)= & -J_{x} \sum_{m, n}|m, n+1\rangle\langle m, n| \\
& -J_{\mathrm{s}} \sum_{m, n} e^{i 2 \pi(\Phi n+\bar{\phi})}|m+1, n\rangle\langle m, n|+\text { H.c. },
\end{aligned}
$$

where in analogy to the relation between the Planck constant $h$ and $\hbar \equiv h /(2 \pi)$, we define $\phi \equiv \phi /(2 \pi)$. We connected these two models by numerically solving the first term in Eq. (B1) to obtain the ground-band Wannier orbitals $w_{0}(x-n a)$ for each lattice site $n$, from which we obtained the longitudinal tunneling strength

$$
\begin{aligned}
J_{x} & =-\int d x w_{0}^{*}(a)\left[-\frac{\hbar^{2}}{2 M_{a}} \frac{d^{2}}{d x^{2}}+\frac{V_{\mathrm{L}}}{2} \cos \left(2 k_{L} \hat{x}\right)\right] w_{0}(0) \\
& =0.066(2) E_{\mathrm{L}} .
\end{aligned}
$$

We then projected the second term in Eq. (B1) to the lowest band subspace giving the synthetic dimension tunneling strength

$$
J_{\mathrm{s}}=\frac{\Omega_{\mathrm{R}}}{2} \int d x\left|w_{0}(x)\right|^{2} e^{2 i k_{R} x}=0.111(3) E_{\mathrm{L}},
$$

where the integral defines a Lamb-Dicke suppression factor, equal to 0.75 for our lattice depth.

\section{APPENDIX C: SIMULATIONS}

All of the numerical simulations presented in the main paper were complete real space 1D discrete variable representation (DVR) simulations of the full light-matter Hamiltonian [46]. Here we compare full-system simulations to those of the reduced Hofstadter model and show: (1) Their qualitative time evolution is the same, but after long times their behavior differs quantitatively, and (2) the predicted peaks in normalized variance as a function of $\Phi$ are indistinguishable. We used a real space DVR method rather than momentum space band structure approach for two reasons: (1) band structure simulations are not possible for irrational flux, since there is no periodic potential, and (2) our observed noise signature depended critically on the spatial extent of our system.

Our simulations of noninteracting atoms included a harmonic potential $V_{\text {ext }}$ that served to define the spatial extent of the ground state wave function

$$
\psi_{\mathrm{HO}}(x) \propto \exp \left[-\frac{1}{2}\left(\frac{x}{\ell_{\mathrm{HO}}}\right)^{2}\right],
$$

with harmonic oscillator length $\ell_{\mathrm{HO}}$. Because the static structure factor $S(q)$ governs the coherence peak width, we selected the frequency of the harmonic potential frequency so the RMS width of $S(q)$ from the numerical simulation was equal to that resulting from $n(x) \propto\left[1-\left(x / R_{\mathrm{TF}}\right)^{2}\right]^{2}$, the $1 \mathrm{D}$ profile derived by integrating a $3 \mathrm{D}$ Thomas-Fermi profile along both transverse directions. This yields the harmonic oscillator length $\ell_{\mathrm{HO}}=20 \sqrt{2 \pi} R_{\mathrm{TF}} / 77 \approx 0.651 R_{\mathrm{TF}}$ giving a spatial density profile with RMS width $w=\ell_{\mathrm{HO}} / \sqrt{2} \approx 0.460 R_{\mathrm{TF}}$. The associated widths in momentum space are $\kappa_{\mathrm{HO}}=1 / \ell_{\mathrm{HO}}$ with a momentum-density RMS width $w_{q}=1 /(2 w)$. With this definition of the potential, every term in both the light matter Hamiltonian and the HH Hamiltonian are fully defined.

We validated our calibrations by performing least squares fits to the data in the middle panel of Fig. 1(b), including $P_{0}(t)$ and $P_{2}(t)$, for a ring-coupling geometry at $\Delta \Phi=-1 / 141$, as well as the data in Fig. 3(d) for the ribbon geometry. The resulting best fit coefficients $\left(\Omega_{\mathrm{R}, 0}, \Omega_{\mathrm{R}, 1}, \Omega_{\mathrm{R}, 2}\right)=(1.04,0.98,0.98) \Omega_{\mathrm{R}}$ and $\left(\delta_{0}, \delta_{1}, \delta_{2}\right)=$ $(0,0,0.02) E_{\mathrm{L}}$ are consistent with the uncertainties of our independent calibrations. All of our simulations include a single phenomenological fitting parameter $\tau=1.5(2) \mathrm{ms}$, obtained only from the fit to the ribbon geometry data, to capture the slow decay of the observed coherent evolution.

We conclude with a side-by-side comparison of the dynamics of these two descriptions, as depicted in Fig. 5, with the full light matter simulation in (a) and the $\mathrm{HH}$ description in (b). Firstly, the time-averaged noise variances, plotted in the first row as a function of $\Phi$, are indistinguishable confirming that our key observation is a property both of our true physical system as well as the reduced $\mathrm{HH}$ model. The second and third rows display the time evolution of the initial state probability $P_{m=1}(t)$ and the noise variance, respectively. These data show that the time evolution of these simulations shares the same dynamical time scale and qualitative features but differ markedly in their quantitative evolution. Still in both cases, 


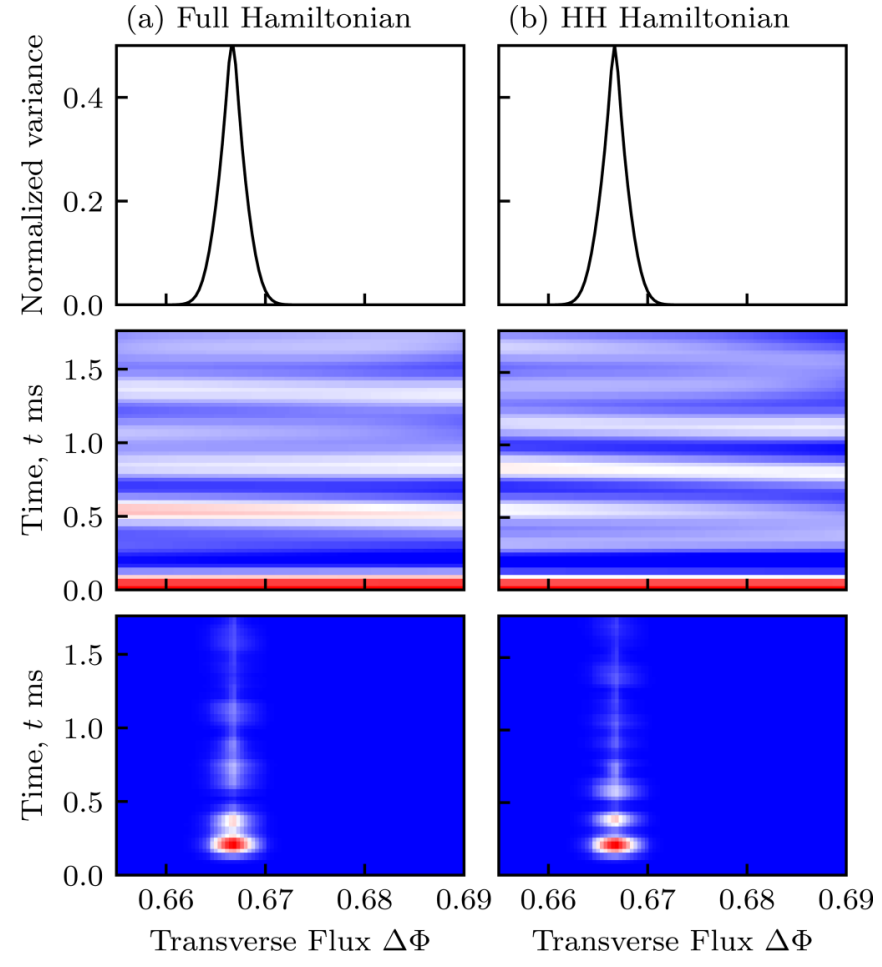

FIG. 5. Full and reduced model comparison. (a) Simulation using the full light matter Hamiltonian and (b) simulation using the reduced $\mathrm{HH}$ Hamiltonian. Both cases modeled a $1.8 \mathrm{~ms}$ evolution time. The first row plots the normalized variance averaged over the evolution time as a function of $\Phi$. The second row plots the occupation probability $P_{m=1}(t)$ of the initial state, as a function of both $\Phi$ and $t$. The third row shows the normalized variance, as a function of both $\Phi$ and $t$.

the probability $P_{m=1}(t)$ shows no feature associated with the peak in the noise variance.

\section{APPENDIX D: AA LATTICES IN MOMENTUM SPACE}

To analytically describe the variance of the time evolution in our experiments, we reduce the $2 \mathrm{D} \mathrm{HH}$ model to a collection of independent 1D AA models. We begin by Fourier transforming the real dimension

$$
|m, n\rangle=\int_{-1 / 2}^{1 / 2} d q e^{-i 2 \pi q n}|m, q\rangle
$$

to realize a series of AA lattices in momentum space:

$$
\begin{aligned}
\hat{H}_{\mathrm{AA}}\left(q_{\mathrm{i}}\right)= & -2 J_{x} \sum_{j} \cos \left[2 \pi\left(q_{\mathrm{i}}+j \Phi\right)\right]\left|j ; q_{\mathrm{i}}\right\rangle\left\langle j ; q_{\mathrm{i}}\right| \\
& -J_{s}\left(e^{i \phi} \sum_{j}\left|j+1 ; q_{\mathrm{i}}\right\rangle\left\langle j ; q_{\mathrm{i}}\right|+\text { H.c. }\right) .
\end{aligned}
$$

In the main text, we approximated irrational transverse fluxes by nearby rational numbers within the finite resolution of the system. Here, we instead approximate rational numbers by nearby irrational numbers. In this case, each AA Hamiltonian $\hat{H}_{\mathrm{AA}}\left(q_{\mathrm{i}}\right)$ describes an infinite chain and the total Hamiltonian $\hat{H}$ sums over an infinite set of such AA chains, where the crystal momentum $q_{\mathrm{i}}$ is written in units of two-photon recoil momenta $2 \hbar k_{\mathrm{L}}$. Here, both the pseudospin and crystal momentum are labeled periodically, namely $|m+M\rangle=|m\rangle$ and $|q+1\rangle=|q\rangle$. Each chain contains a countably infinite set of sites labeled by $\left|j ; q_{\mathrm{i}}\right\rangle \equiv|m, q\rangle=\left|m_{\mathrm{i}}+j, q_{\mathrm{i}}+j \Phi\right\rangle$, where $j \in \mathbb{Z}$. In what follows, we take $m_{\mathrm{i}}=0$ without any loss of generality.

Figure 6 shows an example of an AA chain with $\Phi=$ $P_{\mathrm{r}} / Q_{\mathrm{r}}+\Delta \Phi$, and $\Delta \Phi=1 / 84+(\sqrt{5}-1) / 15000$, close to the simple rational fraction $P_{\mathrm{r}} / Q_{\mathrm{r}}=2 / 3$. This chain is nearly indistinguishable from the corresponding AA ring, with $N_{\mathrm{AA}}=\operatorname{LCM}(M, Q)=84$ sites, where the sites around $j=84$ are near replicas of those around $j=0$. Thus, encircling a rational-flux ring of size $N_{\mathrm{AA}}$ corresponds to traveling between sites of an irrational-flux chain spaced by $N_{\mathrm{AA}}$ sites. We note that even AA rings can contain these near replicas and they are spaced by $N_{\text {rep }}=M /\left[\operatorname{LCM}\left(M, Q_{\mathrm{r}}\right)|\Delta \Phi|\right]$, provided $|\Delta \Phi| \ll \operatorname{LCM}\left(M, Q_{\mathrm{r}}\right)|\Delta \Phi| \lesssim w_{q}$. At long times, beyond those probed in our experiments, we expect to see additional growth of the variance as the atoms travel to the nearest replica, even for the $\phi$-sensitivity suppressed cases at short time scales.

\section{Mean time evolution}

The initial state is a wave packet of width $w_{q}$ in momentum space with initial pseudospin state $\left|m_{\mathrm{i}}=0\right\rangle$ :

$$
|\tilde{\psi}\rangle=\int d q_{\mathrm{i}} \tilde{\psi}_{0}\left(q_{\mathrm{i}}\right)\left|0, q_{\mathrm{i}}\right\rangle
$$

This is unraveled in the AA lattices as

$$
|\tilde{\psi}\rangle=\int d q_{\mathrm{i}} D\left(q_{\mathrm{i}}\right) \sum_{j} \tilde{\psi}_{0, M j}^{\mathrm{AA}}\left(q_{\mathrm{i}}\right)\left|M j ; q_{\mathrm{i}}\right\rangle,
$$

where $\tilde{\psi}_{0, j}^{\mathrm{AA}}\left(q_{\mathrm{i}}\right)=\tilde{\psi}_{0}\left(q_{\mathrm{i}}+j \Phi\right)$ denotes the wave function in the chain containing site $\left|j=0 ; q_{\mathrm{i}}\right\rangle=\left|0, q_{\mathrm{i}}\right\rangle$. The sum over $j$ includes all sites within a chain. $D\left(q_{\mathrm{i}}\right)$ is an everywhere discontinuous function and takes the value of either 1 or 0 , reminiscent of the Dirichlet function, such that the integral over $q_{\mathrm{i}}$ (summing over infinite chains) includes all the momenta within $[0,1)$ without repeating the momenta already connected within a chain.

At time $t$, an initial site $\left|j ; q_{\mathrm{i}}\right\rangle$ evolves to

$$
\left|j(t) ; q_{\mathrm{i}}\right\rangle=\sum_{j^{\prime}} \alpha_{j, j^{\prime}}\left(t ; q_{\mathrm{i}}\right) e^{i \phi\left(j^{\prime}-j\right)}\left|j^{\prime} ; q_{\mathrm{i}}\right\rangle,
$$

where $\alpha_{j, j^{\prime}}\left(t ; q_{\mathrm{i}}\right)=\left\langle j^{\prime} ; q_{\mathrm{i}}\left|\hat{U}_{\mathrm{AA}}\left(t ; q_{\mathrm{i}}\right)\right| j ; q_{\mathrm{i}}\right\rangle$ describes the time evolution from site $j$ to $j^{\prime}$ with $\phi=0$. We can therefore interpret $\alpha_{j, j^{\prime}}\left(t ; q_{\mathrm{i}}\right)$ as the wave function at time $t$ for a particle starting in $\left|j^{\prime} ; q_{\mathrm{i}}\right\rangle$ expressed in the $\left|j ; q_{\mathrm{i}}\right\rangle$ basis, with $\phi=0$.

When $\phi \neq 0$, the time evolution only contributes a phase factor $\phi\left(j^{\prime}-j\right)$, as can be seen from the expansion of the evolution operator:

$$
\hat{U}_{\mathrm{AA}}=e^{-\frac{i \hat{H}_{\mathrm{AA}} t}{\hbar}}=\mathbb{I}-\frac{i t \hat{H}_{\mathrm{AA}}}{\hbar}+\frac{1}{2 !}\left(-\frac{i t \hat{H}_{\mathrm{AA}}}{\hbar}\right)^{2}+\cdots
$$




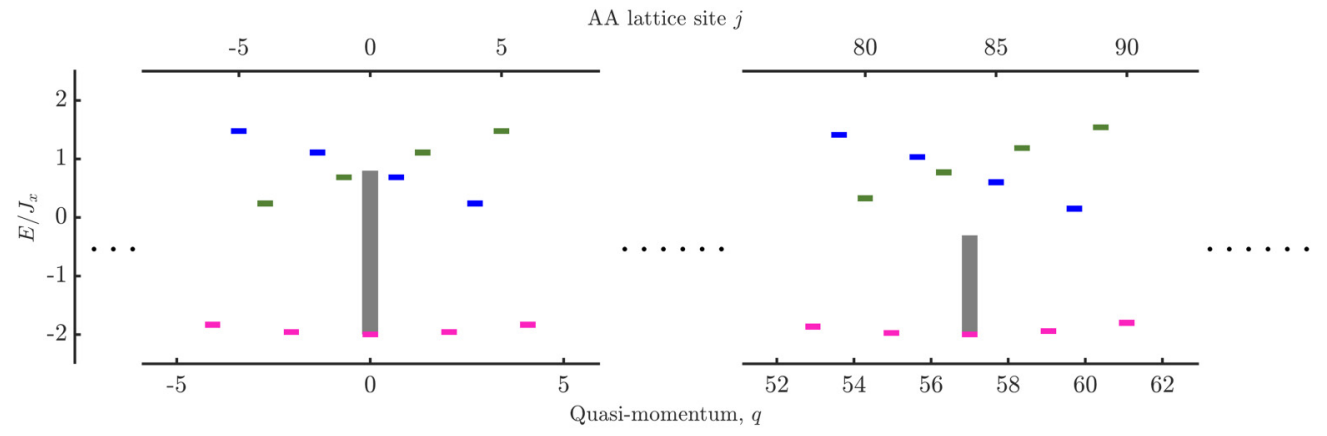

FIG. 6. Irrational flux AA chains. An AA chain with $q_{\mathrm{i}}=0, \Phi=P_{\mathrm{r}} / Q_{\mathrm{r}}+\Delta \Phi$ where $\Delta \Phi=1 / 84+(\sqrt{5}-1) / 15000$ and $P_{\mathrm{r}} / Q_{\mathrm{r}}=2 / 3$. The grey bars show the unraveling of a Gaussian wave packet with width $w_{q}=1 /(2 \pi w)$, where $w=23$.

The initial state has pseudospin $M j_{1}, j_{1} \in \mathbb{Z}$. Consequently, the total wave function of chain $q_{\mathrm{i}}$ at time $t$ evolves to

$$
\left|\tilde{\psi}(t) ; q_{\mathrm{i}}\right\rangle=\sum_{j_{1}, j_{2}} \tilde{\psi}_{0, M j_{1}}^{\mathrm{AA}}\left(q_{\mathrm{i}}\right) \alpha_{M j_{1}, j_{2}}\left(t ; q_{\mathrm{i}}\right) e^{i \phi\left(j_{2}-M j_{1}\right)}\left|j_{2} ; q_{\mathrm{i}}\right\rangle
$$

with the probability to arrive in the final site $\left|j ; q_{\mathrm{i}}\right\rangle$ equal to

$$
\begin{aligned}
\tilde{P}_{j}\left(t ; q_{\mathrm{i}}\right)= & \left|\left\langle j ; q_{\mathrm{i}} \mid \tilde{\psi}(t) ; q_{\mathrm{i}}\right\rangle\right|^{2} \\
= & \sum_{j_{1}, j_{2}} \tilde{\psi}_{0, M j_{1}}^{\mathrm{AA} *}\left(q_{\mathrm{i}}\right) \tilde{\psi}_{0, M j_{2}}^{\mathrm{AA}}\left(q_{\mathrm{i}}\right) \alpha_{M j_{1}, j}^{*}\left(t ; q_{\mathrm{i}}\right) \alpha_{M j_{2}, j}\left(t ; q_{\mathrm{i}}\right) \\
& \times e^{i \phi M\left(j_{1}-j_{2}\right)} .
\end{aligned}
$$

Inserting $\left\langle\exp \left[i \phi M\left(j_{1}-j_{2}\right)\right]\right\rangle_{\phi}=\delta_{j_{1}, j_{2}}$ into the above equation leads to the averaged probabilities:

$$
\left\langle\tilde{P}_{j}\left(t ; q_{\mathrm{i}}\right)\right\rangle_{\phi}=\sum_{j_{1}}\left|\tilde{\psi}_{0, M j_{1}}^{\mathrm{AA}}\left(q_{\mathrm{i}}\right)\right|^{2}\left|\alpha_{M j_{1}, j}\left(t ; q_{\mathrm{i}}\right)\right|^{2} .
$$

This is an incoherent sum over the probabilities of the atoms starting from each initial site weighted by the probability of traveling from that site to the final site.

Assuming the initial wave packet is very narrow, the Hamiltonian in the vicinity of an initially occupied site $\left|j_{1} ; q_{\mathrm{i}}\right\rangle$ is close to that at $\left|j_{1}=0 ; q_{\mathrm{i}}\right\rangle$ and independent of $q_{\mathrm{i}}$. This allows us to replace the evolution from site $j_{1}$ to site $j$ with that from site 0 to $j-j_{1}$, i.e., $\alpha_{j_{1}, j}\left(t ; q_{\mathrm{i}}\right) \rightarrow \alpha_{0, j-j_{1}}(t)$, leading to probability depending only on the distance traveled.

Our experiments measured the probability

$$
\begin{aligned}
\left\langle P_{m_{\mathrm{f}}}(t)\right\rangle_{\phi} & =\int d q_{\mathrm{i}} D\left(q_{\mathrm{i}}\right) \sum_{\ell}\left\langle\tilde{P}_{m_{\mathrm{f}}+M \ell}\left(t ; q_{\mathrm{i}}\right)\right\rangle_{\phi} \\
& \approx \sum_{\ell}\left|\alpha_{0, m_{\mathrm{f}}+M \ell}(t)\right|^{2}
\end{aligned}
$$

to arrive at the final state with pseudospin $m_{\mathrm{f}}+M \ell, \ell \in \mathbb{Z}$, where we made the approximation $\alpha_{j_{1}, j}\left(t ; q_{\mathrm{i}}\right) \rightarrow \alpha_{0, j-j_{1}}(t)$ and reversed the unraveling in Eq. (D2). This equation depends only on the evolution of a particle starting at AA lattice site $|j=0\rangle$. We consider a small range of transverse fluxes in the vicinity of a rational fraction $\Phi_{\mathrm{r}}$ of the flux quantum. Within the range of propagation, the small deviation of the transverse flux is not resolved, i.e., $\alpha_{0, j-j_{1}}^{\Phi}(t) \approx \alpha_{0, j-j_{1}}^{\Phi_{\mathrm{r}}}(t)$. We hereby conclude that the mean time evolution in the vicinity of $\Phi_{\mathrm{r}}$ is a smooth function of $\Phi$.

\section{Variance of the time evolution}

The time evolving variance for arriving in site $m_{f}$ over a uniformly sampled ensemble of Peierls phase $\phi$ is

$$
\operatorname{var}_{m_{\mathrm{f}}}(t) \equiv\left\langle P_{m_{\mathrm{f}}}(t)^{2}\right\rangle_{\phi}-\left\langle P_{m_{\mathrm{f}}}(t)\right\rangle_{\phi}^{2} .
$$

We calculate $\operatorname{var}_{m_{\mathrm{f}}}(t)$ beginning with

$$
\begin{aligned}
\left\langle P_{m_{\mathrm{f}}}^{2}(t)\right\rangle_{\phi}= & \int d q_{1} D\left(q_{1}\right) \int d q_{2} D\left(q_{2}\right) \sum_{\ell_{1}, \ell_{2}}\left\langle\tilde{P}_{m_{\mathrm{f}}+M \ell_{1}}\left(t ; q_{1}\right) \tilde{P}_{m_{\mathrm{f}}+M \ell_{2}}\left(t ; q_{2}\right)\right\rangle_{\phi} \\
\approx & \sum_{j}\left[\int d q_{1} D\left(q_{1}\right) \int d q_{2} D\left(q_{2}\right) \sum_{j_{1}^{\prime}, j_{2}^{\prime}} \tilde{\psi}_{0, M\left(j+j_{1}^{\prime}\right)}^{\mathrm{AA} *}\left(q_{1}\right) \tilde{\psi}_{0, M j_{1}^{\prime}}^{\mathrm{AA}}\left(q_{1}\right) \tilde{\psi}_{0, M\left(-j+j_{2}^{\prime}\right)}^{\mathrm{AA} *}\left(q_{2}\right) \tilde{\psi}_{0, M j_{2}^{\prime}}^{\mathrm{AA}}\left(q_{2}\right)\right] \\
& \times \sum_{\ell_{1}, \ell_{2}}\left[\alpha_{0, m_{\mathrm{f}}+M\left(\ell_{1}-j\right)}^{*}(t) \alpha_{0, m_{\mathrm{f}}+M \ell_{1}}(t) \alpha_{0, m_{\mathrm{f}}+M\left(\ell_{2}+j\right)}^{*}(t) \alpha_{0, m_{\mathrm{f}}+M \ell_{2}}(t)\right],
\end{aligned}
$$

where we have used Eq. (D3) and $\left\langle e^{i M \phi\left(j_{1}+j_{2}\right)}\right\rangle_{\phi}=\delta_{j_{1},-j_{2}}$. The expression in the square bracket in the second line is

$$
=\left|\int d q_{1} \tilde{\psi}_{0}^{*}\left(q_{1}+M \Phi j\right) \tilde{\psi}_{0}\left(q_{1}\right)\right|^{2} \equiv S(M \Phi j),
$$

where $S$ is the static structure factor [47] and the normalization implies $S(0)=1$. We arrived at this expression by reversing the unraveling of the momentum space wave function [noted below Eq. (D2)], i.e., $\int d q_{i} D\left(q_{i}\right) \sum_{j_{i}^{\prime}} \rightarrow \int d q_{i}, i=1,2$. 


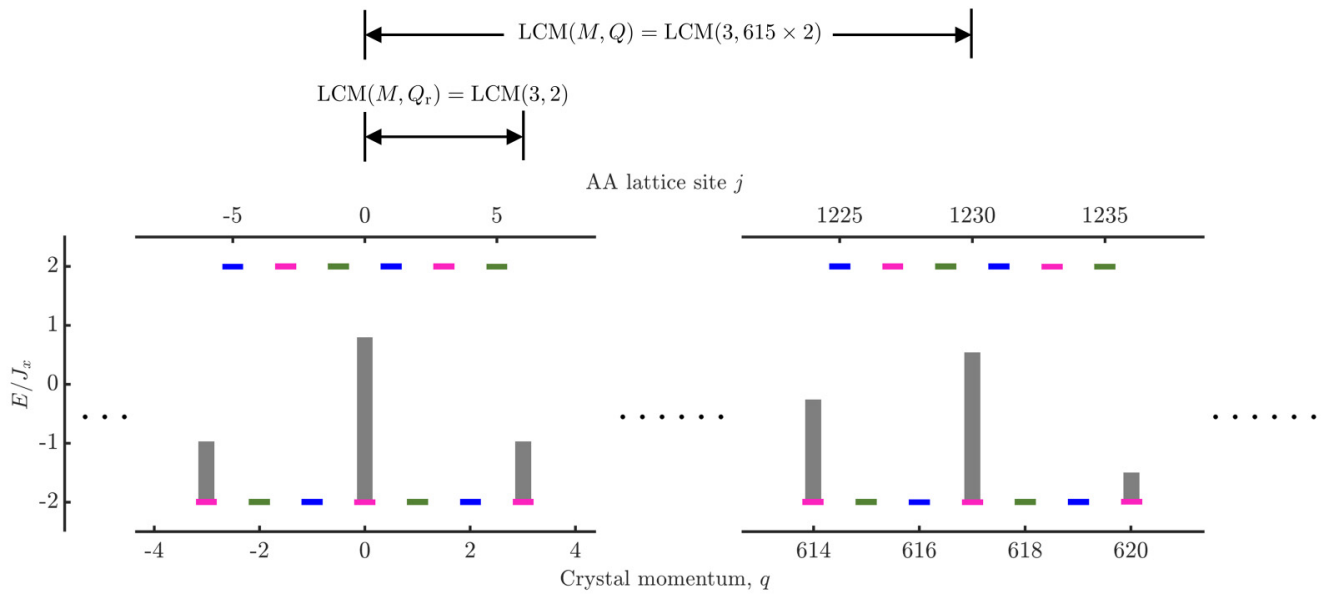

FIG. 7. Irrational flux AA chains with $M \neq \operatorname{LCM}\left(M, Q_{\mathrm{r}}\right)$. An AA chain with $q_{\mathrm{i}}=0, \Phi=P_{\mathrm{r}} / Q_{\mathrm{r}}+\Delta \Phi$ where $\Delta \Phi=1 / 615+(\sqrt{5}-$ 1) $/ 500000$ and $P_{\mathrm{r}} / Q_{\mathrm{r}}=1 / 2$. The gray bars show the unraveling of a Gaussian wave packet with width $w_{q}=1 /(2 \pi w)$, where $w=23$. These parameters were not probed in our experiments.

Combining Eqs. (D5), (D6) we arrive at

$$
\left\langle P_{m_{\mathrm{f}}}^{2}(t)\right\rangle_{\phi} \approx \sum_{j} S(M \Phi j)\left|\sum_{\ell} \alpha_{0, m_{\mathrm{f}}+M(\ell-j)}^{*}(t) \alpha_{0, m_{\mathrm{f}}+M \ell}(t)\right|^{2} .
$$

Importantly, if we also perform a time average we dephase the cross terms in the double sum

$$
\langle\cdots\rangle_{t}=\delta_{j, 0}\left\langle\left[\sum_{\ell}\left|\alpha_{0, m_{\mathrm{f}}+M \ell}(t)\right|^{2}\right]^{2}\right\rangle_{t}+\left(1-\delta_{j, 0}\right) \sum_{\ell}\left\langle\left|\alpha_{0, m_{\mathrm{f}}+M(\ell-j)}(t)\right|^{2}\left|\alpha_{0, m_{\mathrm{f}}+M \ell}(t)\right|^{2}\right\rangle_{t} .
$$

The Kronecker delta functions locate those cases that match conjugated terms with nonconjugated terms, and the last term avoids double counting.

We now shift the first term in the last line to the LHS to get the time-averaged variance

$$
\begin{aligned}
\left\langle\operatorname{var}_{m_{\mathrm{f}}}\right\rangle_{t} & =\sum_{j \neq 0} S(M \Phi j) \sum_{\ell}\left\langle\left|\alpha_{0, m_{\mathrm{f}}+M(\ell-j)}(t)\right|^{2}\left|\alpha_{0, m_{\mathrm{f}}+M \ell}(t)\right|^{2}\right\rangle_{t} \\
\langle\operatorname{var}\rangle_{t} & =\sum_{j \neq 0} S(M \Phi j) \sum_{i}\left\langle\left|\alpha_{0, i-M j}(t)\right|^{2}\left|\alpha_{0, i}(t)\right|^{2}\right\rangle_{t},
\end{aligned}
$$

having used $S(0)=1$. In the second line, we defined the total variance by summing over final states.

Due to the narrow wave-packet assumption, the sites that could possibly contribute to the structure factor $S$ are separated by $\operatorname{LCM}\left(M, Q_{\mathrm{r}}\right)$, rather than $M$ (Fig. 7), where the nearby rational transverse flux $\Phi_{\mathrm{r}}=P_{\mathrm{r}} / Q_{\mathrm{r}}$ with $P_{\mathrm{r}}$ and $Q_{\mathrm{r}}$ being co-prime. In the main text and the experiments, $P_{\mathrm{r}} / Q_{\mathrm{r}}=$ $2 / 3$, while here we derive a general case. Therefore,

$$
\begin{aligned}
S(M \Phi j) & =S[M \Phi j] \delta_{M j, \operatorname{LCM}\left(M, Q_{\mathrm{r}}\right) j} \\
& =S\left[\Delta \Phi_{M, Q_{\mathrm{r}}} j\right] \delta_{M j, \operatorname{LCM}\left(M, Q_{\mathrm{r}}\right) j},
\end{aligned}
$$

where we introduced $\Delta \Phi_{M, Q_{\mathrm{r}}} \equiv \Delta \Phi \operatorname{LCM}\left(M, Q_{\mathrm{r}}\right)$. Only the noninteger part of the variable matters because the crystal momentum $q$ is periodically labeled. If $\left|\Delta \Phi_{M, Q_{\mathrm{r}}}\right| \gg w_{q}$, then the structure factor and hence the variance approach zero for $j \neq 0$. When $\left|\Delta \Phi_{M, Q_{\mathrm{r}}}\right| \lesssim w_{q}$, the closest distance between initially occupied sites on the chain is $\operatorname{LCM}\left(M, Q_{\mathrm{r}}\right)$ (Fig. 7).
This leads to the final form of the total variance

$$
\begin{aligned}
\langle\operatorname{var}\rangle_{t}= & \sum_{j \neq 0} S\left[\Delta \Phi_{M, Q_{\mathrm{r}}} j\right] \\
& \times \sum_{i}\left\langle\left|\alpha_{0, i-\operatorname{LCM}\left(M, Q_{\mathrm{r}}\right) j}(t)\right|^{2}\left|\alpha_{0, i}(t)\right|^{2}\right\rangle_{t} .
\end{aligned}
$$

We now consider the limiting behavior of this function.

(1) For $\left|\Delta \Phi_{M, Q_{\mathrm{r}}}\right| \gg w_{q}$, only $S(0)$ is practically nonzero and we get $\langle\text { var }\rangle_{t}=0$.

(2) For $\left|\Delta \Phi_{M, Q_{r}}\right| \gtrsim w_{q}$, assume that enough time has passed so the wave packets have spread over a range wide compared to the initial distribution, giving

$$
\langle\operatorname{var}\rangle_{t} \approx \sum_{j \neq 0} S\left[\Delta \Phi_{M, Q_{\mathrm{r}}} j\right]\left[\sum_{i}\left\langle\left|\alpha_{0, i}(t)\right|^{4}\right\rangle_{t}\right],
$$

where we made the far-field replacement $\left|\alpha_{0, i-\operatorname{LCM}\left(M, Q_{\mathrm{r}}\right) j}\right|^{2} \approx$ $\left|\alpha_{0, i}\right|^{2}$. Note that $\sum_{i}\left\langle\left|\alpha_{0, i}(t)\right|^{4}\right\rangle_{t}$ is the inverse participation ratio (IPR) in the AA lattice. 
(3) For $\Delta \Phi \rightarrow 0$, we find

$$
\begin{aligned}
\langle\operatorname{var}\rangle_{t}= & \sum_{j, i}\left\langle\left|\alpha_{0, i-\operatorname{LCM}\left(M, Q_{\mathrm{r}}\right) j}(t)\right|^{2}\left|\alpha_{0, i}(t)\right|^{2}\right\rangle_{t}-\sum_{i}\left\langle\left|\alpha_{0, i}(t)\right|^{4}\right\rangle_{t} \\
\approx & \frac{1}{\operatorname{LCM}\left(M, Q_{\mathrm{r}}\right)}-\sum_{i}\left\langle\left|\alpha_{0, i}(t)\right|^{4}\right\rangle_{t} \\
& \rightarrow \frac{1}{\operatorname{LCM}\left(M, Q_{\mathrm{r}}\right)},
\end{aligned}
$$

where we assumed that the wave function is uniform on the scale of $\operatorname{LCM}\left(M, Q_{\mathrm{r}}\right)$ sites (a poor assumption in the insulating phase, and even in our data there is a $\approx 2 \times$ difference in typical averaged populations) and broke the first term into $\operatorname{LCM}\left(M, Q_{\mathrm{r}}\right)$ individual terms $\left|\alpha_{0, i-\operatorname{LCM}\left(M, Q_{\mathrm{r}}\right) j-m}(t)\right|^{2}\left|\alpha_{0, i}(t)\right|^{2}$ with $m$ from 0 to $\operatorname{LCM}\left(M, Q_{\mathrm{r}}\right)-1$. With that we introduced a new sum over $m$, that we used to complete the $j$ sum to be on every lattice site, at which point we used the normalization condition to do this double sum, and the $\rightarrow$ is assuming a spreading wave packet for which the sum of the IPR $i$ will fall to zero.

\section{AA lattices in real space}

To best align with our TOF data, we explained our observations in a momentum space picture. We note that an analogous description in real space can be derived using the dual AA model from that just presented, where the noise suppression for irrational transverse flux is understood in terms of spatial self-averaging. Inserting the Fourier expansion

$$
|m, n\rangle=\frac{1}{\sqrt{M}} \sum_{q_{m}} e^{-i 2 \pi q_{m} m}\left|q_{m}, n\right\rangle,
$$

into the Hamiltonian in Eq. (B2), where $q_{m} \in$ $\left\{0, \frac{1}{M}, \cdots, \frac{M-1}{M}\right\}$, leads to

$$
\begin{aligned}
\hat{H}_{\mathrm{AA}}\left(\bar{\phi}-q_{m}\right)= & -J_{x} \sum_{n}\left(\left|q_{m}, n+1\right\rangle\left\langle q_{m}, n\right|+\text { H.c. }\right) \\
& -2 J_{s} \sum_{n} \cos \left[2 \pi\left(\Phi n+\bar{\phi}-q_{m}\right)\right]\left|q_{m}, n\right\rangle \\
& \times\left\langle q_{m}, n\right|,
\end{aligned}
$$

where $\hat{H}=\sum_{q_{m}} \hat{H}_{\mathrm{AA}}\left(\bar{\phi}-q_{m}\right)$. The original HH model is transformed into $M$ decoupled AA chains in real space, where the quasimomentum $q_{m}$ shifts the phase of the corresponding chain.

Without repeating the analogous derivations as in momentum space, we simply point out that we reach the same results for the mean and variance of the time evolution. One key step of the derivation takes advantage of the displacement property of the Hamiltonian.

The Hamiltonian Eq. (D11) at longitudinal site $n$ is identical to the Hamiltonian at site $n=0$ with the Peierls phase factor changed to $\bar{\phi}^{\prime}=\Phi n+\bar{\phi}$. Expressed in terms of the displacement operator that shifts the longitudinal sites as $|n+\delta n\rangle=\hat{D}(\delta n)|n\rangle$, the displacement property of the Hamiltonian is

$$
\hat{D}(\delta n) \hat{H}(\phi) \hat{D}^{\dagger}(\delta n)=\hat{H}(\phi-\Phi \delta n) .
$$

At irrational fluxes $\Phi$, for a large system, summing over $n$ randomly samples the phases, equivalent to averaging over $\phi$. Therefore, each individual time evolution is expected to evolve as the averaged evolution-a spatial self-averaging effect. On the other hand, at rational fluxes, averaging over $n$ only allows sampling $Q_{\mathrm{r}}$ different phases in each chain and a total of $\operatorname{LCM}\left(M, Q_{\mathrm{r}}\right)$ phases including all chains. For example, at $P_{\mathrm{r}} / Q_{\mathrm{r}}=2 / 3$ and $\phi=0$, only three phases 0 , $2 \pi / 3$, and $4 \pi / 3$ are sampled, inequivalent to averaging over $\phi$. Thus, each time evolution is expected to have its own unique trajectory.

\section{APPENDIX E: SYSTEM SIZE}

We define the relative variance

$$
\sigma\left(\Delta \Phi_{M, Q_{\mathrm{r}}} w\right)=\frac{\langle\operatorname{var}\rangle_{t}}{\left\langle\sum_{m_{\mathrm{f}}}\left\langle P_{m_{\mathrm{f}}}(t)\right\rangle_{\phi}^{2}\right\rangle_{t}} .
$$

Assuming an initial real space Gaussian wave packet,

$$
\psi_{0}(n)=\frac{1}{\pi^{1 / 4} w^{1 / 2}} \exp \left[-\frac{1}{2}\left(\frac{n}{w}\right)^{2}\right],
$$

with width $w$, from its momentum space counterpart, the static structure factor can be obtained by using Eq. (D6)

$$
S(j)=\exp \left[-\frac{1}{2}\left(2 \pi \Delta \Phi_{M, Q_{\mathrm{r}}} w j\right)^{2}\right] .
$$

Along with Eq. (D8) and $\left\langle\sum_{m_{\mathrm{f}}}\left\langle P_{m_{\mathrm{f}}}(t)\right\rangle_{\phi}^{2}\right\rangle_{t} \approx \sum_{i}\left\langle\left|\alpha_{0, i}(t)\right|^{4}\right\rangle_{t}$, this allows us to directly compute the relative variance in the long-evolution time limit

$$
\sigma\left(\Delta \Phi_{M, Q_{\mathrm{r}}} w\right)=\vartheta_{3}\left[0, i 2 \pi\left(\Delta \Phi_{M, Q_{\mathrm{r}}} w\right)^{2}\right]-1
$$

in terms of $\vartheta_{3}(z, \tau)=\sum_{j=-\infty}^{\infty} q^{j^{2}} \eta^{j}$ the third elliptic theta function, where $q=e^{i \pi \tau}$ and $\eta=e^{2 \pi i z}$.

For $\left|\Delta \Phi_{M, Q_{\mathrm{r}}}\right| w \gtrsim 1 / 4$, keeping only the $j=0, \pm 1$ terms, the function is very well described by

$$
\sigma\left(\Delta \Phi_{M, Q_{\mathrm{r}}} w\right) \rightarrow 2 \exp \left[-\frac{1}{2}\left(2 \pi \Delta \Phi_{M, Q_{\mathrm{r}}} w\right)^{2}\right] .
$$

For $\left|\Delta \Phi_{M, Q}\right| w \lesssim 1 / 4$, using one of the Jacobi identities $\vartheta_{3}(z / \tau,-1 / \tau)=\alpha \vartheta_{3}(z, \tau)$, where $\alpha=\sqrt{-i \tau} \exp \left[\pi i z^{2} / \tau\right]$ and keeping only the $j=0$ term, the function is very well described by

$$
\sigma\left(\Delta \Phi_{M, Q_{\mathrm{r}}} w\right) \rightarrow \frac{\sqrt{2 \pi}}{2 \pi\left|\Delta \Phi_{M, Q_{\mathrm{r}}}\right| w}-1 .
$$

However, it is worth noting that this limit requires a very long evolution time to satisfy the approximation made in Eq. (D8), from which we obtained $\sigma\left(\Delta \Phi_{M, Q_{r}} w\right)$. Figure 8 demonstrates the agreement of these two approximate limits with the explicitly evaluated summation and clearly marks the Gaussian wings and enhancement for small $\sigma\left(\Delta \Phi_{M, Q_{\mathrm{r}}} w\right)$. 

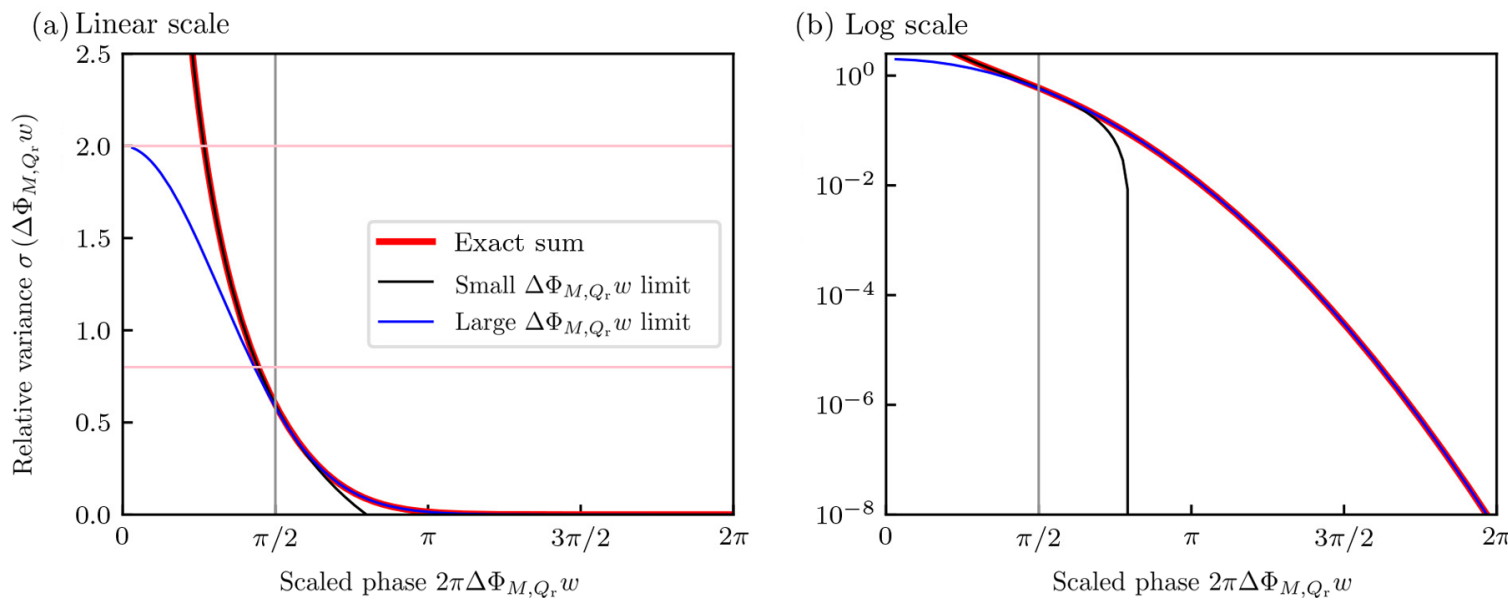

FIG. 8. Time evolution uncertainty plotted in (a) linear and (b) log scale for a Gaussian wave function. In both cases, the thick red curve comes from exactly evaluating Eq. (E1), while the black curve results from the small $w \Delta \Phi_{M, Q_{\mathrm{r}}}$ expansion and the black curve results from the large $w \Delta \Phi_{M, Q_{\mathrm{r}}}$ expansion. The vertical gray line marks $2 \pi w \Delta \Phi_{M, Q_{\mathrm{r}}}=\pi / 2$ where we expect the validity of these expansions to crossover. The pink lines mark the maximum possible noise for quantum states drawn completely at random: The top line at 2 is for $M \rightarrow \infty$, and the bottom line at 0.8 is for $M=3$.

[1] J. Ye, H. J. Kimble, and H. Katori, Quantum state engineering and precision metrology using state-insensitive light traps, Science 320, 1734 (2008).

[2] R. H. Dicke, The effect of collisions upon the Doppler width of spectral lines, Phys. Rev. 89, 472 (1953).

[3] C. Maier, T. Brydges, P. Jurcevic, N. Trautmann, C. Hempel, B. P. Lanyon, P. Hauke, R. Blatt, and C. F. Roos, EnvironmentAssisted Quantum Transport in a 10-Qubit Network, Phys. Rev. Lett. 122, 050501 (2019).

[4] P. G. Harper, The general motion of conduction electrons in a uniform magnetic field, with application to the diamagnetism of metals, Proc. Phys. Soc. London, Sect. A 68, 879 (1955).

[5] D. R. Hofstadter, Energy levels and wave functions of Bloch electrons in rational and irrational magnetic fields, Phys. Rev. B 14, 2239 (1976).

[6] N. Goldman, J. C. Budich, and P. Zoller, Topological quantum matter with ultracold gases in optical lattices, Nat. Phys. 12, 639 (2016).

[7] N. R. Cooper, J. Dalibard, and I. B. Spielman, Topological bands for ultracold atoms, Rev. Mod. Phys. 91, 015005 (2019).

[8] H. Miyake, G. A. Siviloglou, C. J. Kennedy, W. C. Burton, and W. Ketterle, Realizing the Harper Hamiltonian with LaserAssisted Tunneling in Optical Lattices, Phys. Rev. Lett. 111, 185302 (2013).

[9] M. Aidelsburger, M. Atala, M. Lohse, J. T. Barreiro, B. Paredes, and I. Bloch, Realization of the Hofstadter Hamiltonian with Ultracold Atoms in Optical Lattices, Phys. Rev. Lett. 111, 185301 (2013).

[10] C. R. Dean, L. Wang, P. Maher, C. Forsythe, F. Ghahari, Y. Gao, J. Katoch, M. Ishigami, P. Moon, M. Koshino et al., Hofstadter's butterfly and the fractal quantum Hall effect in moiré superlattices, Nature (London) 497, 598 (2013).

[11] B. Stuhl, H.-I. Lu, L. Aycock, D. Genkina, and I. Spielman, Visualizing edge states with an atomic Bose gas in the quantum Hall regime, Science 349, 1514 (2015).
[12] M. Mancini, G. Pagano, G. Cappellini, L. Livi, M. Rider, J. Catani, C. Sias, P. Zoller, M. Inguscio, M. Dalmonte et al., Observation of chiral edge states with neutral fermions in synthetic Hall ribbons, Science 349, 1510 (2015).

[13] J. H. Han, J. H. Kang, and Y. Shin, Band Gap Closing in a Synthetic Hall Tube of Neutral Fermions, Phys. Rev. Lett. 122, 065303 (2019).

[14] C.-H. Li, Y. Yan, S. Choudhury, D. B. Blasing, Q. Zhou, and Y. P. Chen, A Bose-Einstein condensate on a synthetic Hall cylinder, arXiv:1809.02122.

[15] C. Hainaut, I. Manai, J.-F. Clément, J. C. Garreau, P. Szriftgiser, G. Lemarié, N. Cherroret, D. Delande, and R. Chicireanu, Controlling symmetry and localization with an artificial gauge field in a disordered quantum system, Nat. Commun. 9, 1382 (2018).

[16] X.-W. Luo, J. Zhang, and C. Zhang, Tunable flux through a synthetic Hall tube of neutral fermions, Phys. Rev. A 102, 063327 (2020).

[17] R. B. Laughlin, Quantized Hall conductivity in two dimensions, Phys. Rev. B 23, 5632 (1981).

[18] L. Taddia, E. Cornfeld, D. Rossini, L. Mazza, E. Sela, and R. Fazio, Topological Fractional Pumping with Alkaline-EarthLike Atoms in Synthetic Lattices, Phys. Rev. Lett. 118, 230402 (2017).

[19] Summing the phase factors around the tube gives $\Phi_{\mathrm{L}}=$ $M[\Phi n+\phi /(2 \pi)] ; \Phi_{\mathrm{L}}$ depends on longitudinal position $n$ owing to the contribution $2 \pi i \Phi n$ from the transverse flux.

[20] E. J. Meier, F. A. An, A. Dauphin, M. Maffei, P. Massignan, T. L. Hughes, and B. Gadway, Observation of the topological Anderson insulator in disordered atomic wires, Science 362, 929 (2018).

[21] The Thomae function is zero for irrational $\Phi$ and $1 / Q$ for rational $\Phi$. Our analysis [Eq. (D9)] shows that the expected structure is proportional to a modified Thomae function that becomes $1 / \operatorname{LCM}(\mathrm{Q}, \mathrm{M})$ for rational $\Phi$.

[22] Y.-J. Lin, A. R. Perry, R. L. Compton, I. B. Spielman, and J. V. Porto, Rapid production of ${ }^{87} \mathrm{Rb}$ Bose-Einstein condensates in 
a combined magnetic and optical potential, Phys. Rev. A 79, 063631 (2009).

[23] Y. Castin and R. Dum, Bose-Einstein Condensates in Time Dependent Traps, Phys. Rev. Lett. 77, 5315 (1996).

[24] D. Trypogeorgos, A. Valdés-Curiel, N. Lundblad, and I. B. Spielman, Synthetic clock transitions via continuous dynamical decoupling, Phys. Rev. A 97, 013407 (2018).

[25] R. P. Anderson, D. Trypogeorgos, A. Valdés-Curiel, Q.-Y. Liang, J. Tao, M. Zhao, T. Andrijauskas, G. Juzeliūnas, and I. B. Spielman, Realization of a deeply subwavelength adiabatic optical lattice, Phys. Rev. Research 2, 013149 (2020).

[26] The position-space Raman matrix elements $\propto e^{i\left(2 k_{\mathrm{R}} x+\phi\right)}|m+1, x\rangle\langle m, x|$, sampled on a lattice with positions $x=n a$, yield the phase factor in Eq. (1), with $\Phi=k_{\mathrm{R}} / k_{\mathrm{L}}$.

[27] D. L. Campbell and I. B. Spielman, Rashba realization: Raman with rf, New J. Phys. 18, 033035 (2016).

[28] I. B. Spielman, W. D. Phillips, and J. V. Porto, Mott-Insulator Transition in a Two-Dimensional Atomic Bose Gas, Phys. Rev. Lett. 98, 080404 (2007).

[29] S. Aubry and G. André, Analyticity breaking and Anderson localization in incommensurate lattices, Ann. Israel Phys. Soc. 3, 18 (1980).

[30] M. Kohmoto, Metal-Insulator Transition and Scaling for Incommensurate Systems, Phys. Rev. Lett. 51, 1198 (1983).

[31] R. Zhang, Y. Yan, and Q. Zhou, Localization on a synthetic Hall cylinder, arXiv:2012.02204.

[32] S. N. Evangelou and J.-L. Pichard, Critical Quantum Chaos and the One-Dimensional Harper Model, Phys. Rev. Lett. 84, 1643 (2000).

[33] For a 1D chain of three sites, population is maximally transferred out of the initial state at $t=\pi \hbar /\left(3 J_{\mathrm{s}}\right)$ with periodic boundaries and $t=\pi \hbar /\left(2 \sqrt{2} J_{\mathrm{s}}\right)$ with hard-wall boundaries.

[34] M. V. Berry and D. H. J. O'Dell, Ergodicity in wave-wave diffraction, J. Phys. A: Math. Gen. 32, 3571 (1999).

[35] A. Petrescu and K. Le Hur, Chiral Mott insulators, meissner effect, and Laughlin states in quantum ladders, Phys. Rev. B 91, 054520 (2015).
[36] M. Calvanese Strinati, E. Cornfeld, D. Rossini, S. Barbarino, M. Dalmonte, R. Fazio, E. Sela, and L. Mazza, Laughlin-Like States in Bosonic and Fermionic Atomic Synthetic Ladders, Phys. Rev. X 7, 021033 (2017).

[37] F. Kozarski, D. Hügel, and L. Pollet, Quasi-one-dimensional hall physics in the Harper-Hofstadter-Mott model, New J. Phys. 20, 043001 (2018).

[38] X. Zhou, G. Chen, and S. Jia, Synthetic Hall tube of interacting fermions, Phys. Rev. A 102, 043313 (2020).

[39] M. Buser, C. Hubig, U. Schollwöck, L. Tarruell, and F. Heidrich-Meisner, Interacting bosonic flux ladders with a synthetic dimension: Ground-state phases and quantum quench dynamics, Phys. Rev. A 102, 053314 (2020).

[40] L. D. Turner, K. F. E. M. Domen, and R. E. Scholten, Diffraction-contrast imaging of cold atoms, Phys. Rev. A 72, 031403(R) (2005).

[41] A. R. Perry, S. Sugawa, F. Salces-Carcoba, Y. Yue, and I. B. Spielman, Multiple-camera defocus imaging of ultracold atomic gases, arXiv:2102.08292.

[42] A. Valdés-Curiel, D. Trypogeorgos, Q. Y. Liang, R. P. Anderson, and I. B. Spielman, Topological features without a lattice in Rashba spin-orbit coupled atoms, Nat. Commun. 12, 593 (2021).

[43] L. J. LeBlanc, M. Beeler, K. Jimenez-Garcia, A. R. Perry, S. Sugawa, R. Williams, and I. B. Spielman, Direct observation of zitterbewegung in a Bose-Einstein condensate, New J. Phys. 15, 073011 (2013).

[44] A. Valdés-Curiel, D. Trypogeorgos, Q.-Y. Liang, J. Tao, M. Zhao, I. B. Spielman (unpublished).

[45] P. L. Kapitza and P. A. M. Dirac, The reflection of electrons from standing light waves, Math. Proc. Cambridge Philos. Soc. 29, 297 (1933).

[46] D. T. Colbert and W. H. Miller, A novel discrete variable representation for quantum mechanical reactive scattering via the S-matrix Kohn method, J. Chem. Phys. 96, 1982 (1992).

[47] M. P. Marder, Condensed Matter Physics (John Wiley \& Sons, New York, NY, 2010). 